\title{
La décroissance comme projet urbain et paysager
}

\section{Serge Latouche}

\section{OpenEdition}

Journals

Édition électronique

URL : http://journals.openedition.org/edl/507

DOI : $10.4000 /$ edl. 507

ISSN : 2296-5084

\section{Éditeur}

Université de Lausanne

\section{Édition imprimée}

Date de publication : 15 mai 2013

Pagination : 259-274

ISBN : 978-2-940331-32-1

ISSN : 0014-2026

\section{Référence électronique}

Serge Latouche, "La décroissance comme projet urbain et paysager », Études de lettres [En ligne], 1-2 I 2013, mis en ligne le 15 mai 2016, consulté le 18 décembre 2020. URL : http:// journals.openedition.org/edl/507; DOI : https://doi.org/10.4000/edl.507 


\section{LA DÉCROISSANCE COMME PROJET URBAIN ET PAYSAGER}

Pour comprendre l'approche urbaine et paysagiste de la décroissance, il importe d'abord de saisir comment la société de croissance engendre un désastre territorial, puis de voir les implications architecturales et urbanistiques du projet de la décroissance face à une crise de civilisation. Si la crise est politique, le remède doit l'être aussi. C'est la raison pour laquelle le projet de la décroissance passe nécessairement par une refondation du politique. La ville décroissante devrait être une ville à empreinte écologique réduite, entretenant un rapport étroit avec l'écosystème (une bio-région). La bio-région ou écorégion peut être définie comme une entité spatiale cohérente traduisant une réalité géographique, sociale et historique. Le mouvement des villes en transition est peutêtre la forme de construction par le bas de ce qui se rapproche le plus d'une société de décroissance.

On peut partir du paradoxe de l'urbanisme contemporain, c'est-à-dire du contraste entre la quantité des architectes (et aussi des urbanistes) d'excellente qualité (y compris dans le champ de l'habitat écologique) et le désastre urbain et paysager actuel dans lequel le monde est enfermé.

L'exposition "habiter écologique" ${ }^{1}$ à laquelle j'ai participé constitue un bon exemple de cette qualité évoquée. A la génération des grands, Le Corbusier, Ludwig Mies van der Rohe (1886-1969), Walter Gropius (1883-1969), dont certains avaient déjà des préoccupations écologiques, comme Franck Lloyd Wright (1867-1959), Alvar Aalto (1898-1976) et Hassan Fathy (1900-1989), a fait suite une nouvelle génération tout aussi riche (Oscar Niemeyer [1907-2012], Lucio Costa [1902-1998], Ricardo Bofill, Renzo Piano, Jean Prouvé, Jean Nouvel, Frank Gehry

I. "Quelles architectures pour une ville durable?», Cité de l'architecture et du patrimoine, Paris, 13 mai- $1^{\text {er }}$ novembre 2009. 
et naturellement Massimiliano Fuksas), mais aussi des spécialistes de l'habitat écologique comme Philippe Madec, Christian de Portzamparc, Jacques Ferrier, et une pléthore de jeunes talentueux.

Souvent très séduisante à l'unité (les villas isolées en particulier ou les bâtiments de prestige), cette architecture est dans l'ensemble très décevante, à la fois parce qu'elle échoue à «faire ville» et surtout, parce qu'elle a globalement échoué à empêcher la décomposition du tissu urbain, la "cimentification» du territoire, le mitage du paysage, la montée de la laideur du cadre de vie et la destruction de l'environnement, sans parler de l'échec à réduire la consommation d'énergie et l'empreinte écologique. Tout en s'efforçant d'y porter remède, ces architectes et urbanistes ont été cependant les complices du désastre.

Désastre urbain, c'est le constat du grand architecte portugais, Alvaro Siza:

[...] le plus grave est la dévastation du territoire, le ratage de cette discipline qu'est l'utilisation de la terre [...]. Nous assistons à la fin d'un ordre des choses qui préfigure peut-être autre chose, que nous ne connaissons pas encore. Et sans doute était-ce inévitable. Mais dans l'immédiat, la qualité est marginale et nous sommes devant un désastre ${ }^{2}$.

Platon déclarait que les murs mêmes de la cité éduquent le citoyen. Dans La cité du soleil, Tomaso Campanella avait pensé cette forme d'éducation de façon systématique: toute l'histoire des sciences était représentée sur les murs des sept enceintes de la ville... Mais à quoi peuvent bien éduquer les murs de nos villes et de nos banlieues avec leur urbanisme le plus souvent laid et sans âme, et une publicité agressive et omniprésente? Au mieux, ils ne peuvent former que des consommateurs et usagers frustrés, au pire des «sauvageons" rebelles. Ils ne contribueront pas à façonner les personnalités fortes et indépendantes capables de résister à la manipulation médiatique et à la propagande politique devenue son sous-produit.

Pour comprendre l'approche urbaine et paysagiste de la décroissance, il importe d'abord de saisir comment la société de croissance engendre un désastre territorial, puis de voir les implications architecturales et urbanistiques du projet de la décroissance.

2. A. Siza, "A cet âge, on est radical», "At that age, one is radical», p. 52. 


\section{Le désastre urbain de la société de croissance}

Le désastre urbain et paysager, que tout un chacun peut constater, est le résultat de logiques qui échappent bien évidemment aux architectes et aux urbanistes. Toutefois, ceux-ci en sont les complices en même temps qu'ils cherchent à y porter remède. Nous sommes en face d'une forme de schizophrénie. Nous vivons encore dans la ville "productiviste", pensée et structurée en fonction de l'automobile sous des formes prétendument rationnelles - il suffit de penser à la cité radieuse de Le Corbusier -, avec sa ségrégation des espaces, ses zones industrielles, ses quartiers résidentiels sans $v^{3}{ }^{3}$. Marinetti, en 1909, dans le Manifeste du mouvement futuriste, anticipant le projet de Le Corbusier de raser Paris, veut détruire Venise au nom du progrès:

Détournez le cours de canaux pour inonder les musées! [...] A vous les pioches et les marteaux! Sapez les fondements des villes vénérables! ${ }^{4}$

Ceauşescu l'a réalisé à Bucarest, Pompidou est mort trop tôt pour achever le projet de Le Corbusier sur Paris, mais Bruxelles est devenu l'exemple du massacre conjoint de la spéculation et de la modernisation.

On a pu parler à juste titre de la destruction des villes en temps de paix ${ }^{5}$ avec l'éclatement des centres anciens et la spéculation immobilière effrénée qui rejettent les couches inférieures et moyennes dans les périphéries, la prolifération des centres commerciaux, l'extension de zones pavillonnaires, l'émergence de tours, l'éventrement par les autoroutes et la multiplication des "non-lieux», gares, aéroports, hypermarchés et l'asphyxie de la circulation automobile ${ }^{6}$. Il s'agit là d'un des symptômes d'une crise plus vaste engendrée par la sur- ou hyper-modernité (terme plus juste, à nos yeux, que la "post-modernité»).

C'est le triomphe de la laideur. Déjà en 1972, Bernard Charbonneau, écologiste français et précurseur de la décroissance, dénonçait dans $L a$ fin du paysage ${ }^{7}$ (titre de son livre illustré) les retombées négatives du productivisme sur le paysage et l'environnement.

3. Y. Cochet, Antimanuel d'écologie, p. 247.

4. F.-T. Marinetti, Manifeste du futurisme, p. 1.

5. J.-C. Michea, L'enseignement de l'ignorance et ses conditions modernes.

6. Voir sur ce point les analyses de Marc Augé et de Marco Revelli.

7. B. Charbonneau, La fin du paysage. 
La modernité, avec l'industrialisation du XIX ${ }^{\mathrm{e}}$ siècle, avait détruit la ville médiévale et baroque, engendrant des problèmes (et des souffrances énormes) dont témoignent les romans de Dickens ou de Zola; toutefois un certain équilibre s'était maintenu ou reconstitué autour des grands boulevards (l'exemple du Paris de Haussman est emblématique). Si elle n'avait pas maintenu un rapport respectueux avec le paysage (il suffit de penser aux mines de charbon, de fer et aux autres ravages industriels), la catastrophe était encore relativement limitée du fait que l'humanité ne dépassait pas deux milliards d'individus et que l'industrialisation ne touchait que quelques pays.

Cet équilibre tout relatif traduisait dans le tissu urbain un équilibre tout aussi relatif entre la société avec sa moralité traditionnelle (éthique du travail, sens du devoir, de l'honneur et de l'honnêteté) et ses institutions (armée, justice, éducation, beaux arts) et l'économie capitaliste de l'accumulation illimitée. La rupture de cet équilibre a été consommée avec ce qu'on appelle la "globalisation» ou "mondialisation" et que l'on peut dater de manière symbolique de la chute du mur de Berlin en 1989. Ce n'est pas l'extension des échanges ou de la finance à l'échelle planétaire qui est nouvelle (celle-ci existe au moins depuis 1492), c'est l'inverse, la marchandisation et la financiarisation du monde. Avec les trois $\mathrm{D}$, déréglementation de la société salariale et de l'Etat social, désintermédiation financière, décloisonnement des économies et des marchés, décidés en 1986 par Ronald Reagan et Margaret Thatcher, on assiste littéralement à l'«omnimarchandisation» du monde. Tout devient objet de trafic, jusqu'au corps humain, au sang, aux gènes. On passe d'une société avec marché à une société de marché, d'une société avec croissance à une société de croissance qui peut être définie comme une société dominée par une économie de croissance et qui tend à s'y laisser absorber. La croissance pour la croissance devient ainsi l'objectif primordial sinon le seul de la vie. Le cancer de la Croissance (avec un C majuscule) ne détruit pas seulement la ville, il lacère le territoire, ronge le sens des lieux et détricote le tissu social.

On assiste à «l'explosion de l'urbain», selon l'expression de la sociologue Tiziana Villani ${ }^{8}$. Il s'agit d'un processus d'artificialisation de la vie. L'homme prétend recréer le monde mieux que Dieu et que la nature. Les OGM, les nanotechnologies, le clonage, l'élevage industriel de poissons,

8. T. Villani, «La décroissance à l'âge de la révolution urbaine», p. 58. 
etc., en sont une illustration. L'aboutissement serait le "cyberman", l'homme artificiel. Désormais, le résultat le plus visible est la transformation du monde réel, celui dans lequel nous sommes condamnés à vivre en décharge et en poubelle. La faillite de Dubaï et de sa tour de $800 \mathrm{~m}$ inoccupée constitue un symbole de la faillite du rêve américain et de son urbanisme. Le projet de tour de $1 \mathrm{~km}$ de haut ne sera probablement jamais construit. La ville productiviste appartient au passé, mais la destruction du monde se poursuit.

Les remèdes ne sont pas à la hauteur du défi. Déjà au XIXe siècle, en réaction au cancer de l'industrialisation, sont apparus des utopies socialistes avec des implications urbaines. Le phalanstère de Charles Fourier par exemple contient une inscription urbaine du projet, le palais social, qui a directement inspiré la réalisation du «familistère de Guise» (en Lorraine) de Jean Baptiste Godin (1817-1888). Victor Considérant, disciple de Fourier, écrivait en 1834:

L'architecture est l'art central, c'est l'art qui résume tous les autres, et par conséquent, résume la société elle-même9

Il a existé aussi des "utopies urbaines», c'est-à-dire des projets qui prétendaient remédier aux problèmes sociaux et environnementaux grâce à l'organisation urbaine. Ainsi, les cités-jardins ou "Garden cities of tomorrow" d'Ebenezer Howard (1850-1928). Celui qui a poussé le plus loin la sensibilité paysagère est sans doute William Morris avec son livre News of Nowhere ${ }^{10}$, où il préconise une désindustrialisation de l'Angleterre et la restauration des paysages. Concrètement il s'est battu pour protéger les arbres sur les berges de la Tamise.

Toutefois, l'architecture éco-responsable avec l'habitat bioclimatique n'est pas la solution, au mieux constitue-t-elle un hypothétique élément de solution. La "ville soutenable" préconisée par la charte d'Aalborg (1994) est plus une forme de modernisation écologique du capitalisme ("greenwashing») qu'un véritable remède au désastre du productivisme. Sous les termes d' "habitats groupés", de "covoisinages", de "cohabitats", d'« éco-quartiers", de "villes écologiquement soutenables ", différents projets ont été réalisés en Europe et aux Etats-Unis.

9. V. Considérant, Description du phalanstère et considérations sociales sur l'architectonique, p. 34.

Io. W. Morris, Nouvelles de nulle part. 
Les réalisations les plus connues et les plus abouties, apparues vers la fin des années 2000, sont celles du quartier Vauban à Fribourg-en-Brisgau (Allemagne), Houten (périphérie d'Utrecht, 40'000 ha) et de Bedzed ("Beddington zero energy développement») dans la ville de Sutton au sud de Londres. En fin de compte, ce sont des îlots de soutenabilité dans une mer de pollution urbaine qu'ils ne réussiront pas à transformer. La faillite et l'échec retentissants des "éco-villes" chinoises sont symptomatiques. Les rares projets, lancés en fanfare comme à Chongming, sont dans l'impasse. L'éco-ville de Dongtan à Chongming en face de Shanghai a été promue à marche forcée en 2006-2008 pour servir de vitrine écologique à l'Exposition universelle, mais le parrain du projet a été limogé en 2008 pour corruption et le projet mal conçu a été abandonné. Les autres projets (Huangbaiyu et Tianjin) sont mal en point. L'économie l'emporte sur l'écologie ${ }^{11}$. Dans ces projets il s'agit d'habiter mieux mais non de changer le rapport à la nature, au paysage et à la logique consumériste.

Les tentatives honorables des urbanistes de remédier à la crise urbaine en proposant des schémas ingénieux - régions urbaines, cités jardins, ville totale, réseaux urbains, conurbation (Geddes), Broadacre city (Wright) - recherchant une nouvelle articulation ville/campagne sont vouées à l'échec à défaut d'une analyse globale de la faillite de la société de croissance.

Le fonctionnalisme formalisé par la Charte d'Athènes de Le Corbusier (1943) qui prétendait lutter contre le "désordre urbain" a engendré finalement un désordre plus grand au prix d'une explosion de l'empreinte écologique de la ville. Selon la prophétie de Lewis Mumford, la megapolis se transforme en tyrannopolis, puis finit en nekropolis ${ }^{12}$. Tel semble être le destin de l'hyperpolis virtuelle constituée par la finance et les média mondialisés.

Si la crise est politique, le remède doit l'être aussi. C'est la raison pour laquelle le projet de la décroissance passe nécessairement par une refondation du politique et partant, de la polis, de la cité et de son rapport avec la nature. Le projet urbain est nécessairement second par rapport au projet sociétal et le projet architectural par rapport au projet

II. Ph. Grangereau, "Avec ses écovilles, la Chine joue aux échecs».

I2. Th. Paquot, Terre urbaine et Utopies et utopistes. 
urbain. Le "désastre» urbain n'est pas le résultat d'une défaillance des architectes ni des urbanistes, mais celui d'une crise de civilisation.

Naturellement, le désastre urbain est moins visible dans les centres villes (même celui des villes détruites et reconstruites, suivant la vision de Le Corbusier, comme Stuttgart), que dans les périphéries et les "villettopoli» abandonnées par les urbanistes. Le tissu local et urbain ne peut être recomposé que par la réalisation d'une société de décroissance.

\section{Le projet de la décroissance et ses implications urbaines et paysagistes}

Pour pouvoir esquisser ce que pourrait être l'urbanisme et l'architecture dans une société de décroissance, il faut préciser le sens du projet de la société de décroissance et en voir les implications urbaines et paysagistes.

Qu'est-ce que la décroissance? Le mot d'ordre de décroissance vise avant tout à souligner fortement la nécessité d'abandonner le projet insensé du développement pour le développement, de la croissance pour la croissance.

Bien évidemment, il ne s'agit pas d'un renversement caricatural qui consisterait à prôner la décroissance pour la décroissance. En particulier, la décroissance n'est pas la croissance négative. On sait que le simple ralentissement de la croissance plonge nos sociétés dans le désarroi en raison du chômage et de l'abandon des programmes sociaux, culturels et environnementaux qui assurent un minimum de qualité de vie. On peut imaginer quelle catastrophe serait un taux de croissance négatif! De même qu'il n'y a rien de pire qu'une société travailliste sans travail, il n'y a rien de pire qu'une société de développement sans développement. Rigoureusement parlant, il faudrait parler d'a-croissance (comme on dit a-théisme) plutôt que de décroissance. Il s'agit précisément de l'abandon d'une foi et d'une religion: celle de l'économie.

Le changement réel de perspective, nécessaire pour construire une société autonome de décroissance, peut être conçue à travers l'utopie radicale, systématique et ambitieuse des huit « $\mathrm{R}$ »: Réévaluer, Reconceptualiser, Restructurer, Relocaliser, Redistribuer, Réduire, Réutiliser, Recycler. Ces huit objectifs interdépendants sont susceptibles d'enclencher un cercle vertueux de décroissance sereine, conviviale 
et soutenable ${ }^{13}$. Il ne s'agit pas encore d'un programme puisqu'on est au niveau de la conception, mais on peut facilement en déduire des propositions concrètes pour la réalisation.

On peut dire des huit «R» qu'ils sont tous plus importants les uns que les autres. Il me semble, cependant, que trois d'entre eux ont un rôle stratégique: la réévaluation, parce qu'elle commande tout changement, la réduction parce qu'elle condense tous les impératifs pratiques de la décroissance, et la relocalisation parce qu'elle concerne la vie quotidienne et l'emploi de millions de gens ${ }^{14}$. La problématique de la ville et du territoire détruits et à repenser s'inscrit dans ce contexte plus vaste du monde lacéré, de la perte des repères et de la crise du local. Le désastre urbain s'accompagne d'un désastre rural et de la destruction des paysages. Toutefois, dans l'optique de la construction d'une société de décroissance sereine, la relocalisation n'est pas seulement économique. La politique, la culture, le sens de la vie doivent retrouver leur ancrage territorial. Le mot clef est "autonomie».

La relocalisation occupe donc une place centrale dans l'utopie concrète et féconde de la décroissance et se décline presque immédiatement en programme politique. La décroissance semble ainsi rénover la vieille formule des écologistes: penser globalement, agir localement. Relocaliser l'économie et la vie est une condition indépassable de la soutenabilité. Si l'utopie de la décroissance implique une pensée globale, on ne peut la réaliser aujourd'hui qu'en partant des territoires. Il s'agit de «reterritorialiser » (selon l'expression d'Alberto Magnaghi ${ }^{15}$ ), retrouver un site et le re-habiter.

Alors, à quoi ressemblera la ville décroissante? La ville décroissante devrait être une ville à empreinte écologique réduite, entretenant un rapport étroit avec l'écosystème (une bio-région). Plutôt que de songer à construire des villes nouvelles, ce sera d'abord une autre manière d'habiter la ville, au Nord comme au Sud. La ville consomme de la

I3. On pourrait allonger la liste des « $\mathrm{R}$ » avec: radicaliser, reconvertir, redéfinir, redimensionner, remodeler, repenser, etc., mais tous ces " $\mathrm{R}$ » sont plus ou moins inclus dans les huit premiers.

I4. "Quatre thèmes peuvent structurer l'espace en devenir des sociétés de sobriété: l'autosuffisance locale et régionale, la décentralisation géographique des pouvoirs, la relocalisation économique et le protectionnisme, la planification concertée et le rationnement» (Y. Cochet, Pétrole apocalypse, p. 208).

I5. A. Magnaghi, Le projet local. 
basse entropie (énergie, ressources, aliments, etc.) et exporte massivement de la haute entropie (déchets, pollution). Il s'agit d'un prédateur écologique qui consomme une surface "fantôme" très supérieure à sa seule superficie:

Pour qu'un mètre carré de surface urbaine fonctionne dans les villes espagnoles, il faut 60 mètres carrés d'espace rural, de sol agricole, forêt ou prairie, pour permettre aux troupeaux de produire les biens et services réclamés par les grandes villes. L'empreinte écologique urbaine n'arrête pas de croître. Il y a 50 ans, les villes n'avaient besoin pour chaque mètre carré que de $25 \mathrm{~m}^{2}$ de campagne. Si on fait une projection, à ce train là, en 2050, il faudra $500 \mathrm{~m}^{2}$ de sol rural par $\mathrm{m}^{2}$ urbanisé. L'empreinte écologique du citadin espagnol représente 4 fois l'empreinte soutenable (6 ha 395/1, 8) ${ }^{16}$.

Plus la ville est étalée, "fonctionnelle» (Le Corbusier), plus cette empreinte est importante. Ce qui ne veut pas dire qu'il faille verticaliser... Les tours sont des gouffres d'énergie et n'accroissent pas vraiment la densité. Il faut certainement réinventer une ville plus «compacte». L'habitat individualisé, isolé, même écologiquement bien pensé, est une hérésie urbanistique, du point de vue de la décroissance, car des hectares de terres agricoles disparaissent chaque année sous le béton et le bitume. La construction groupée et le logement collectif présentent une efficacité énergétique plus poussée. A la place des mégapoles actuelles il faut imaginer une ville écologique, faite de villages urbains où cyclistes et marcheurs utiliseront une énergie renouvelable. Dans la ville "décroissante", les habitants retrouveront ainsi le plaisir de flâner cher à Baudelaire ou Walter Benjamin. Réapprendre à habiter le monde est donc un impératif!

On peut songer à organiser des bio-régions urbaines. La bio-région ou écorégion peut être définie comme une entité spatiale cohérente traduisant une réalité géographique, sociale et historique. Elle peut être plus ou moins rurale ou urbaine - distinction malheureusement en voie de disparition. La bio-région urbaine, constituée d'un ensemble complexe de systèmes territoriaux locaux doté d'une forte capacité à l'autosoutenabilité écologique, vise à la réduction des déséconomies externes et de la consommation d'énergie.

I6. E. Reyes, «Decrecimiento y medio urbano", p. 57. 
Politiquement, une bio-région urbaine pourrait être conçue comme une municipalité de municipalités ou "une ville de villes» voire une "ville de villages », bref un réseau polycentrique ou multipolaire ${ }^{17}$. Selon l'idée de l'anarcho-écologiste Murray Bookchin, il s'agit de considérer une aire métropolitaine comme une articulation de quartiers autonomes fonctionnant en tant que communes juxtaposées. Yona Friedman écrit ainsi que:

La ville qui, depuis des siècles. a fonctionné suivant la formule: «endroit où tout s'échange", va devenir une arche de Noé destinée à assurer la survie de l'espèce malgré le déluge. Une très grande autonomie, une très grande autarcie vont donc lui être nécessaires [... ${ }^{18}$.

Cette autonomie ne signifie pas pour autant une autarcie complète. On peut faire du commerce avec les régions qui ont fait le même choix et "laissé tomber" le productivisme. On recherchera aussi l'autonomie énergétique locale: les énergies renouvelables «sont adaptées aux sociétés décentralisées, sans grandes concentrations humaines. Mais cette dispersion est aussi un avantage: chaque région du monde possède un potentiel naturel pour développer une ou plusieurs filières d'énergie renouvelable» ${ }^{19}$.

Sera-t-on capable un jour d'habiter poétiquement les tours de bureaux, les stades, les échangeurs, les centres commerciaux, les décharges et tous les parcs d'attractions, tout ce que l'architecte hollandais Rem Koolhaas appelle le «junkspace» ${ }^{20}$.

La réponse vient peut-être de Yona Friedman: «[...] pour transformer le mal en bien, nous devrons nous débarrasser du conditionnement que nous avons subi ${ }^{21}$. Il s'agit d'habiter autrement la même ville: penser le Paris/le pari de la décroissance.

17. A. Magnaghi, «Dalla città metropolitana alla (bio)regione urbana».

I8. Y. Friedman, L'architecture de survie, p. 164.

19. Y. Cochet, Pétrole apocalypse, p. 140.

20. "Le junkspace”, c'est ce qui reste quand la modernisation est à bout de course, ou, plutôt ce qui se coagule au fur à mesure qu'elle se fait» (Ch. Laurens, "Se rappeler que la Terre est belle», p. 145).

2I. Y. Friedman, L'architecture de survie, p. 143. 
Dans un premier temps, la ville décroissante, ce peut être la ville actuelle d'où auront été éliminées la publicité, les voitures et la grande distribution et où auront été introduits des jardins partagés, des pistes cyclables, une gestion en régie des biens communs (eau, services de base) et développé le cohabitat et les ateliers de quartier. Une reconversion sera nécessaire mais aussi une certaine désindustrialisation, dont le résultat, réalisé grâce à des équipements sophistiqués mais conviviaux, serait la preuve qu'il est possible de produire autrement. Même si la part auto-produite n'est pas totale, elle est importante ${ }^{22}$.

Dans son beau livre, Manifesto per la felicità. Come passare dalla società del ben-avere a quella del ben-essere, Stefano Bartolini présente ainsi la ville relationnelle, qui correspond presque exactement au projet de la décroissance:

La ville relationnelle constitue un aspect clef de ma proposition de faire aux enfants une place bien plus importante qu'à l'heure actuelle parce qu'ils constituent le paradigme du lien étroit entre l'espace et la mobilité qu'il faudra instaurer dans l'expérience relationnelle. Les enfants doivent disposer d'espaces piétonniers de qualité près de la maison et de la possibilité d'y arriver tout seuls.

Les éléments clefs d'une ville relationnelle sont: restriction drastique de l'espace accordé à la voiture particulière pour faire en sorte que tous les citadins utilisent les transports publics; forte densité d'habitation; abondance de places, de parcs, d'îlots piétonniers de qualité, de centres sportifs, etc.; les aires piétonnes idéales devraient être près de la mer, d'un lac, d'une rivière, d'un ruisseau, d'un canal; elles devraient traverser la ville de manière à former un réseau piétonnier et cyclable; il devrait y avoir des trottoirs spacieux et des pistes cyclables; de grands espaces de propriété publique devraient entourer la ville pour y construire des parcs et des maisons ${ }^{23}$.

Et pour le Sud? Il faut partir de la réalité. Deux milliards de gens vivent dans des bidonvilles ou des favelas autoconstruites et n'accèderont jamais à la ville productiviste. La vision de Yona Friedman de l'architecture et de l'urbanisme de survivance est certainement plus réaliste pour le Sud

22. I. Granstedt, Du chômage à l'autonomie conviviale, p. 70. A la fin de sa vie, André Gorz a développé des idées proches (voir A. Gorz, "Crise mondiale, décroissance et sortie du capitalisme»).

23. S. Bartolini, Manifesto per la felicità, p. 42. 
et d'ailleurs en cohérence avec la ville décroissante au Nord. La ville pauvre est faite d'un ensemble de «bidonvillages». Selon Friedman:

Le bidonvillage est la société anarchiste des pauvres et il ne s'agit pas là d'un choix idéologique ou politique; ce type de société s'est constitué simplement parce que l'expérience avait prouvé qu'il était celui qui assurait, au bidonvillage, les meilleures chances de survie ${ }^{24}$.

Finalement:

La réponse de l'architecture de survie aux problèmes courants serait donc: construire moins, mais apprendre à habiter autrement; exploiter moins nos champs, mais par contre, apprendre à réviser nos critères de la "comestibilité»; vivre dans les villes où nous vivons, mais nous organiser avec moins de circulation et vivre dans notre village urbain, isolé des autres villages urbains, éloignés de nous par la distance ${ }^{25}$.

En attendant les nécessaires changements de "gouvernance» mondiale, et l'arrivée au pouvoir de gouvernements nationaux acquis à l'objection de croissance, de nombreux acteurs locaux ont implicitement ou explicitement emprunté la voie de l'utopie féconde de la décroissance.

Si le projet local contient d'évidentes limites, ne sous-estimons pas les possibilités d'avancées de la politique à ce niveau. On peut mentionner «la rete del nuovo municipio, la rete delle città lente (slow cities), le città in transizione (transition towns), le città post carbone ${ }^{26}$, les nombreuses expériences de villes "vertueuses» comme l'expérience de la commune de Mouans Sartoux sous l'impulsion de son maire André Aschieri ${ }^{27}$,

24. Y. Friedman, L'architecture de survie, p. 110.

25. Ibid., p. 141.

26. En italien dans le texte. "La Rete del Nuovo Municipio»: réseau d'autorités locales territoriales, de chercheurs et d'associations qui favorise des nouvelles formes de démocratie participative pour un développement locale soutenable. «La Rete delle città lente» : réseau des villes du bien vivre. "Transition towns»: villes en transition, visant à assurer la résilience de la communauté face au pic pétrolier et au dérèglement climatique. "Città post carbone» (ou "Post carbon cities/communities») : villes énergétiquement autonomes.

27. Réouverture de la gare et de la desserte ferroviaire à contre courant, multiplication des mises en régies pour les "biens publics communs" (l'eau, les transports et jusqu'aux pompes funèbres), développement de pistes cyclables et des espaces verts, maintien des agriculteurs locaux et des petits commerces, refus de la spéculation immobilière et de l'installation de grandes surfaces, ont permis d'éviter une "périphérisation " considérée 
l'expérience de Correns, ce village du Var où tous les vignerons ont décidé de passer à l'agriculture biologique, ou celle de $\mathrm{Barjac}^{28}$. Dans ce dernier exemple, on voit comment l'introduction de produits bio dans les cantines scolaires, décidée par un maire courageux et créatif, peut de proche en proche modifier en profondeur la totalité de la vie d'un village. Tout cela relié à des initiatives plus petites comme les $\mathrm{GAS}^{29}$ ou les AMAP ${ }^{30}$, etc. Le mouvement des villes en transition né en Irlande (Kinsale près de Cork) et qui s'épanouit en Angleterre (à Totnes) est peut-être la forme de construction par le bas de ce qui se rapproche le plus d'une société de décroissance. Ces villes, selon la charte du réseau, visent d'abord à l'autosuffisance énergétique en prévision de la fin des énergies fossiles et plus généralement à la résilience. Ce concept emprunté à la physique en passant par l'écologie scientifique peut être défini comme la permanence qualitative du réseau d'interactions d'un écosystème, ou, plus généralement, comme la capacité d'un système à absorber les perturbations et à se réorganiser en conservant essentiellement ses fonctions, sa structure, son identité et ses rétroactions ${ }^{31}$.

La résilience désigne plus simplement la capacité d'un écosystème à résister à des changements de son environnement. Comment, par exemple, de grandes agglomérations urbaines vont-elles pouvoir affronter la fin du pétrole, l'élévation de la température et toutes les catastrophes prévisibles? La réponse de l'expérience écologique est que si la spécialisation permet d'accroître les performances dans un domaine, elle fragilise la résilience de l'ensemble. La diversité au contraire renforce la résistance et les capacités d'adaptation. Réintroduire les jardins potagers, la polyculture, l'agriculture de proximité, de petites unités artisanales, multiplier les sources d'énergie renouvelable renforcent donc la résilience.

Pour conclure, on peut reprendre deux citations d'architectes. La première d'Enrico Frigerio :

comme inéluctable il y a 30 ans et ont redonné du sens au vivre local dont le festival annuel du livre est un vibrant symbole.

28. Devenue célèbre par le film de Jean-Paul Jaud, Nos enfants nous accuseront.

29. GAS - Groupe d'Achat solidaire.

30. AMAP - Association pour le Maintien d'une Agriculture Paysanne.

3I. R. Hopkins, The Transition Handbook. 
Je crois que le type de l'architecte esthète, créateur de formes nouvelles, est aujourd'hui anachronique ${ }^{32}$.

La seconde de Yona Friedman:

Après tout, nous sommes peut-être en train de redécouvrir qu'assurer sa propre survie peut être aussi la Fête ${ }^{33}$.

Au total, la ville décroissante, premier pas vers une société d'abondance frugale, préservera l'environnement qui est en dernier ressort la base de toute vie, ouvrira pour chacun un accès plus démocratique à l'économie, réduira le chômage, renforcera la participation (et donc l'intégration) et aussi la solidarité, fortifiera la santé des citadins grâce à la croissance de la sobriété et à la diminution du stress. Même si ce n'est pas l'objet d'une politique spécifique, l'impact sur le paysage, sera nécessairement positif.

Serge Latouche

Université Paris-Sud (Orsay)

32. E. Frigerio, Slow Architecture, p. 101. «L'architetto esteta, creatore di forme, credo sia oggi quasi anacronistico ». Traduction française de l'auteur.

33. Y. Friedman, L'architecture de survie, p. 169. 


\section{BIBLIOGRAPHIE}

Bartolini, Stefano, Manifesto per la felicità. Come passare dalla società del ben-avere a quella del ben-essere, Roma, Donzelli, 2010.

Charbonneau, Bernard, La fin du paysage, Paris, Anthropos, 1972.

Cochet, Yves, Pétrole apocalypse, Paris, Fayard, 2005.

—, Antimanuel d'écologie, Paris, Bréal, 2009.

Considérant, Victor, Description du phalanstère et considérations sociales sur l'architectonique, Paris, Guy Durier, 1979 (1848).

Friedman, Yona, L'architecture de survie. Où s'invente aujourd'hui le monde de demain, Paris, Castermann, 1978.

Frigerio, Enrico, Slow Architecture. Istruzioni per l'uso, Melfi, Libria, 2009 (Mosaico).

Gorz, André, "Crise mondiale, décroissance et sortie du capitalisme», Entropia, 2 (2007): Décroissance et travail, p. 51-59.

Grangereau, Philippe, "Avec ses écovilles, la Chine joue aux échecs", Libération, 17 mai 2010. URL: http://www.liberation.fr/ terre/0101635793-avec-ses-ecovilles-la-chine-joue-aux-echecs.

Granstedt, Ingmar, Du chômage à l'autonomie conviviale, Lyon, A plus d'un titre, 2007 (1982).

Hopkins, Rob, The Transition Handbook. From Oil Dependency to Local Resilience, Foxhole/Dartington/Totnes/Devon, Green Books Ltd, 2008.

Laurens, Christophe, "Se rappeler que la Terre est belle», Entropia, 8 (2010): Territoires de la décroissance, p. 137-146.

Magnaghi, Alberto, Le projet local, Sprimont (Belgique), Pierre Mardaga, 2003.

—, «Dalla città metropolitana alla (bio)regione urbana », in Il progetto di territorio nella città metropolitana, a cura di Anna Marson, Firenze, Alinea, 2006, p. 69-112.

Marinetti, Filippo Tommaso, Manifeste du futurisme, Le Figaro, 20 février 1909.

Michea, Jean-Claude, L'enseignement de l'ignorance et ses conditions modernes, Paris, Climats, 1999. 
Morris, William, Nouvelles de nulle part, Paris, Aubier bilingue, 2004. Paquot, Thierry, Terre urbaine. Cinq défis pour le devenir urbain de la planète, Paris, La Découverte, 2006.

—, Utopies et utopistes, Paris, La Découverte, 2007.

ReYes, Eugenio, «Decrecimiento y medio urbano. Tres discursos sobre el decrecimiento de las ciudades", in Decrecimientos. Sobre lo que hay que cambiar en la vida cotidiana, éd. par Carlos Taibo, Madrid, Catarata, 2010, p. 57.

SizA, Alvaro, "A cet âge, on est radical ", "At that age, one is radical», entretien par Dominique Machabert avec Fernando Tàvora, Alvaro Siza, Gonçalo Byrne, Eduardo Souto de Moura et Joao Alvaro Rocha, Techniques et Architecture, 466 (2003), p. 50-54.

Villani, Tiziana, "La décroissance à l'âge de la révolution urbaine: écologie politique et hyperpolis", Entropia, 8 (2010): Territoires de la décroissance, p. 58-67. 